\title{
CDISC Diabetic Kidney Disease Therapeutic Area User Guide Version 1.0
}

National Cancer Institute

\section{Source}

National Cancer Institute. CDISC Diabetic Kidney Disease Therapeutic Area User Guide Version 1.0. NCI Thesaurus. Code C161439.

The 1.0 version of the CDISC Diabetic Kidney Disease therapeutic area user guide. 